\title{
Multiaxial behavior of foams - Experiments and modeling
}

\author{
Laurent Maheo ${ }^{1,2, a}$, Sandra Guérard ${ }^{3, b}$, Gérard Rio $^{2, \mathrm{c}}$, Adrien Donnard ${ }^{3, \mathrm{~d}}$, and Philippe Viot ${ }^{3, \mathrm{e}}$ \\ ${ }^{1}$ Ecoles de Saint-Cyr Coëtquidan, CREC, 56381 Guer, France \\ ${ }^{2}$ Université de Bretagne Sud, LIMATB, 56100 Lorient, France \\ ${ }^{3}$ Arts et Metiers ParisTech, Campus de Bordeaux-Talence, I2M, 33405 Talence, France
}

\begin{abstract}
Cellular materials are strongly related to pressure level inside the material. It is therefore important to use experiments which can highlight (i) the pressure-volume behavior, (ii) the shear-shape behavior for different pressure level. Authors propose to use hydrostatic compressive, shear and combined pressure-shear tests to determine cellular materials behavior. Finite Element Modeling must take into account these behavior specificities. Authors chose to use a behavior law with a Hyperelastic, a Viscous and a Hysteretic contributions. Specific developments has been performed on the Hyperelastic one by separating the spherical and the deviatoric part to take into account volume change and shape change characteristics of cellular materials.
\end{abstract}

\section{Introduction}

Foams and more generally cellular materials are specifically used as filling material in sandwich structures with a function of energy dissipation. Their ability to resist to impact and shock, combined with their low density, now make them ideal candidates for passive safety and energy dissipation [1].

Foam behavior has been studied by authors in numerous publications according to simple characterization tests that allow obtaining input parameters for the models proposed by industrial codes [2]. Classically, only uniaxial compressive experiments are needed to feed the numerical model. Even if the numerical models succeed in describing foam behavior under uniaxial loading conditions, they are not well adapted to represent multiaxial behavior occurring under complex loadings, such as punch impacts. To improve mechanical description of foams, Hanssen et al. [3] proposed several experiments. One of this test which is very important to describe foam behavior is the hydrostatic compressive test [4] and some numerical models take into account the behavior under hydrostatic pressure conditions [2].

Aware of the importance of hydrostatic pressure on foams behavior, other authors have developed hydrostatic compressive [4] and multi-axial tests [5]. These tests are performed to achieve a representation of a load surface (or behavior transition) of foams in the equivalent stress plane as a function of hydrostatic stress. Other representation that takes into account, independently, loading direction characterized by the Lode angle, hydrostatic pressure and stress intensity has also been used recently and seems to be a good way to describe cellular materials [5].

\footnotetext{
a e-mail: laurent.maheo@univ-ubs.fr

b e-mail: sandra.guerard@ensam.eu

${ }^{c}$ e-mail: gerard.rio@univ-ubs.fr

d e-mail: adrien.donnard@ensam.eu

e e-mail: philippe.viot@ensam.eu
}

In this scientific framework, authors decided to investigate experimentally, and numerically, cellular materials behavior. Foam behavior will be described numerically using a generalist yet powerful phenomenological model, namely HVH (Hyperelastic, Viscous, Hysteretic) model [6]. The HVH model has been enhanced to take into account foam specificities such as volume change and shape change sensitivities, and the coupling behavior between the volume change and the shape change behaviors. Experimental study has been led to get mechanical characteristics of the numerical model and to guide the choice for the numerical developments. Shear tests have therefore been performed to get shape change behavior. Shear tests under different level of pressure can be possible to identify the coupling between pressure and shear by using an original hexapod device that allows combined loadings, such as uniaxial compression/tension and shear tests. Digital Image Correlation technique has been used to analyze the area of the specimen that is subjected to shear behavior and obtain the shear strain field. The stress is calculated thanks to a 3-dimensional sensor. Experimental results clearly show that shear behavior is related to the level of hydrostatic pressure. Numerical model has therefore been improved accordingly and shows good agreement to describe foam behavior under multiaxial loading conditions.

\section{Experiments}

Every tests presented is this paper have been performed on a polypropylene (PP) foam, named Arpro ${ }^{(C)}$ and produced by JSP society. The density of the foam is between 90 and $100 \mathrm{~kg} \cdot \mathrm{m}^{3}$.

\subsection{Uniaxial compressive test}

Uniaxial compressive tests can be performed in quasistatic conditions using an electromechanical device or in 


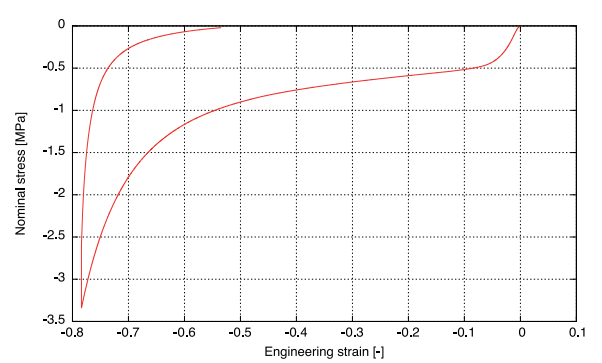

Figure 1. Classical compressive test on a PP foam. Evolution of the nominal compressive stress versus the engineering compressive strain.

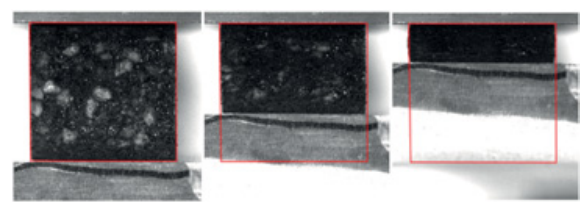

Figure 2. Classical compressive test on a PP foam. Foam specimen for engineering strain values of $0.00,0.35$ and 0.70 .

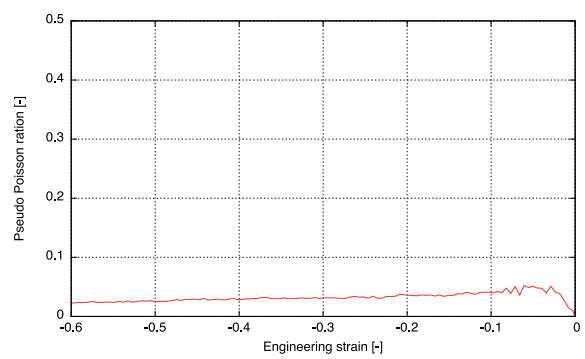

Figure 3. Classical compressive test on a PP foam. Evolution of the pseudo Poisson's ratio versus the engineering compressive strain.

dynamic conditions using a flywheel device or a SplitHopkinson Pressure Bar device as in [7-9]. Classical foam behavior is observed in Fig. 1 with a first elastic stage, a second stress plateau stage and a third densification stage. Nominal stress is classical calculated: $\sigma_{n o m}=\frac{F_{z}}{S_{0}}$ where $F_{z}$ is the force along the $z$-axis and $S_{0}$ is the cross section area.The engineering strain is calculated as: $\varepsilon_{\text {eng }}=\frac{h-h_{0}}{h_{0}}$ with $h$ and $h_{0}$ the current and the initial height of the sample, respectively.

It can also be noticed in Fig. 2 that the transversal displacement of the foam under uniaxial compressive loading conditions is quite small even for large transformations.

In this context of large transformations a pseudo Poisson's ratio $v_{p}$ can be calculated using image analysis. Every images obtained during the test is analyzed with an Image $^{(C)}$ procedure. (i) An area is defined at the center of the foam specimen and related to its height. (ii) A threshold and an outline macro give the outlines of the specimen. (iii) The specimen width over the initial width ratio is calculated for every image using a mean of the position of each specimen side. The pseudo Poisson's ratio can be calculated as: $v_{p}=-\frac{l-l_{0}}{l_{0}} \frac{h_{0}}{h-h_{0}}$ with $l$ and $l_{0}$ the current and the initial width, respectively. Its evolution can be displayed versus the engineering compressive strain in Fig. 3. It can be observed that during the compressive test, the pseudo Poisson's ratio is about 0.03 .

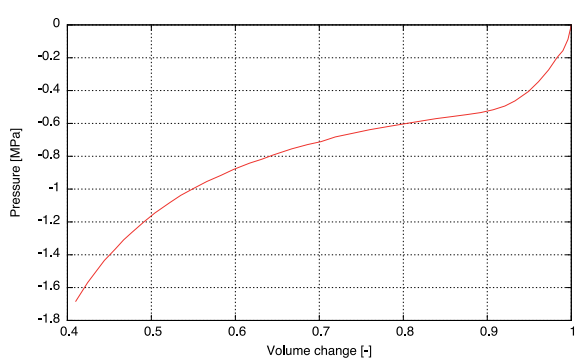

Figure 4. Hydrostatic compressive test on a PP foam. Evolution of the pressure versus the volume change.

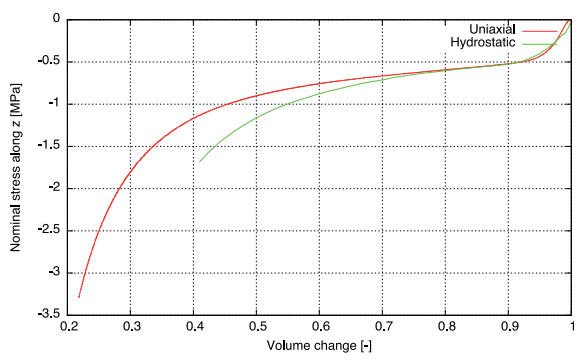

Figure 5. Comparison of uniaxial and hydrostatic compressive tests on a PP foam. Evolution of the $\sigma_{z}$ versus the volume change.

\subsection{Hydrostatic compressive test}

Hydrostatic compressive tests on PP foam in quasi-static and dynamic conditions have already been performed by Viot and published in [4]. The results are presented Fig. 4.

The shape of the hydrostatic curve looks like the one of the uniaxial compressive test. For this reason, it seems interesting to plot both curves of the uniaxial and hydrostatic compressive tests on the same figure. With the assumption of a null-pseudo Poisson's ratio, the relation between the engineering strain $\varepsilon_{\text {eng }}$ and the volume variation $V$ is: $V=1-\varepsilon_{\text {eng }}$. If the results are presented with $\sigma_{z}$ vs. $V$, Fig. 5, it can be noticed that the hydrostatic behavior is quite the same than the uniaxial compressive behavior. The level of the stress plate is almost the same and is approximately equal to 0.5-1.0 MPa. At the beginning of both tests, before a volume change of $\varepsilon_{z}=0.7$, the difference between two tests does note exceed $10 \%$. This shows the importance of the volume change contribution on the foam behavior.

\subsection{Shear test}

\subsubsection{Discussion about specimen dimensions}

In order to evaluate the shape change contribution, shear tests are needed. However, if we want to exhibit the influence of the pressure on the shear behavior, combined compressive-shear experiments should be used. With these considerations, the shape of foam specimen must allow to perform combined compressive-shear tests using an hexapod device, see Figs. 6 and 7.

The Hexapod device allows to perform three translations and three rotations. An additional seventh electromechanical jack can be independently used to improve compressive velocity but has been locked for this study. A three-force sensor is located at the top of the foam specimen in order to record the force vector during 


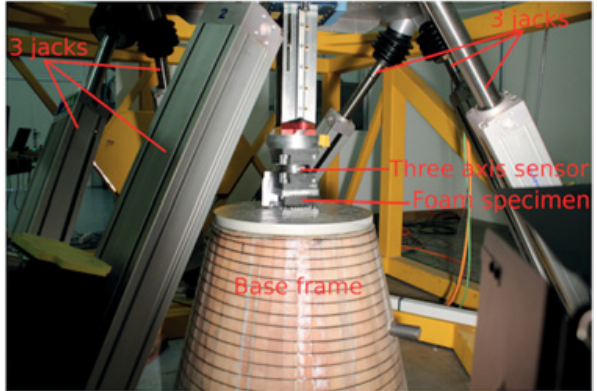

Figure 6. Hexapod device using for combined shear-compressive tests.

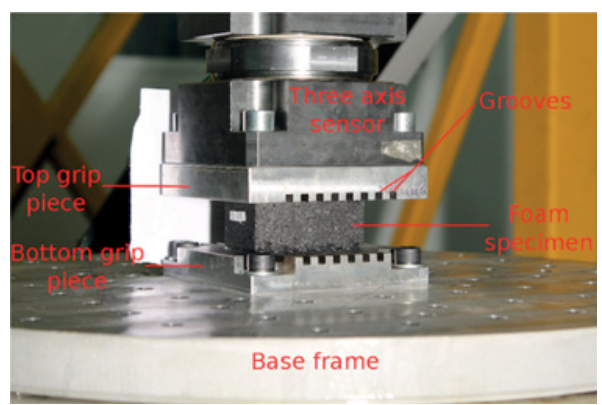

Figure 7. Foam specimen before a combined shear-compressive test.

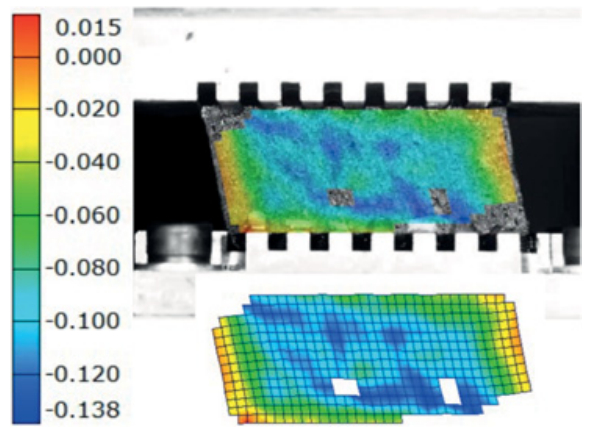

Figure 8. Digital Image Correlation Technique used for a shear test. Shear strain $\varepsilon_{x y}=\frac{\gamma_{x y}}{2}$ field just before the failure.

the test. The foam specimen is glued on two grip pieces. The top one is screwed on the force sensor and the bottom one is screwed on a base frame which is screwed in the ground. In order to improve the efficiency of the grips, $5 \mathrm{~mm}$-grooves have been machined on the grips and on the specimen foam. The dimensions of foam specimen are $75 \times 30 \times 40 \mathrm{~mm}^{3}$ in length, height and depth, respectively. These dimensions do not guarantee pure shear test as mentioned by Bouvier et al. [10]. Indeed, some flexural phenomena occur at both side of the specimen and can not be neglected. However this test can give some first needed informations.

\subsubsection{Shear test results}

Three quasi-static shear tests have been performed at a strain rate of $\dot{\gamma}_{x y}=10^{-2} \mathrm{~s}^{-1}$ until a strain level of $\gamma_{y x}^{\max }=1$. Digital Image Correlation technique has been used to measure strain on the specimen using a zone of interest of 128 pixels (see Fig. 8). It can be seen two areas

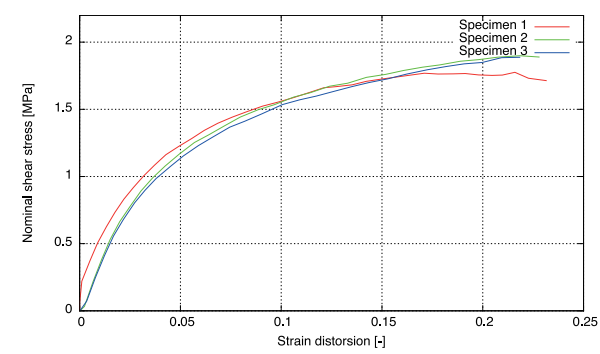

Figure 9. Shear behavior with the evolution of the shear stress $\tau_{x y}$ vs. the strain distorsion $\gamma_{x y}$ until the failure.

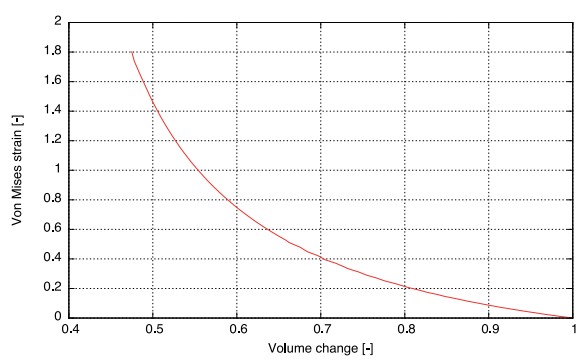

Figure 10. Evolution of the shape change vs. the volume change during an unixial compressive test with a pseudo Poisson's ration of $v=0.03$.

on this figure. The first one is the area which is located at both side of the specimen and is subjected to flexion behavior. The size of this area decreases with the height of the specimen. The second one is a non-homogeneous strain field area which is located at the center of the specimen. This non-homogeneity is due to foam beads with different densities which involve localization of deformation [9]. A average of strain field can be performed in this area to get the mean strain distorsion. The failure occurs for the three tested specimen at a strain level of $\gamma_{y x}^{\text {mean }}=0.224 \pm 0.06$.

Using the measurements of the three-axis force sensor, it can be possible to plot the evolution of the shear stress vs. the strain distorsion (see Fig. 9).

It can be shown that the results are quite repeatable and the shear stress reach a value of $2 \mathrm{MPa}$, higher than the stress value plateau for hydrostatic and uniaxial compressive test. Aware of the presence of flexion behavior on both side of the specimen, future tests on specimen with an adapted geometry need to be performed.

\subsection{Experiments summary}

In order to sum up the three previous experiments using only one diagram, authors decided to plot the evolution of the principal stress $\sigma_{I}$ vs. two independent variables:

- the volume change $V$,

- the shape change using the Von Mises strain $\varepsilon_{V M}=$ $\sqrt{\frac{3}{2} \overline{\bar{\varepsilon}}_{D}: \overline{\bar{\varepsilon}}_{D}}$ where $\overline{\bar{\varepsilon}}_{D}$ is the deviatoric strain tensor.

Hydrostatic compressive test and shear test assume that no shape change and no volume change occur during each test, respectively. Thanks to the measurement of transversal strain during the uniaxial compressive test, i.e. $v=0.03$, it is possible to plot the evolution of the shape change vs. the volume change, see Fig. 10. 


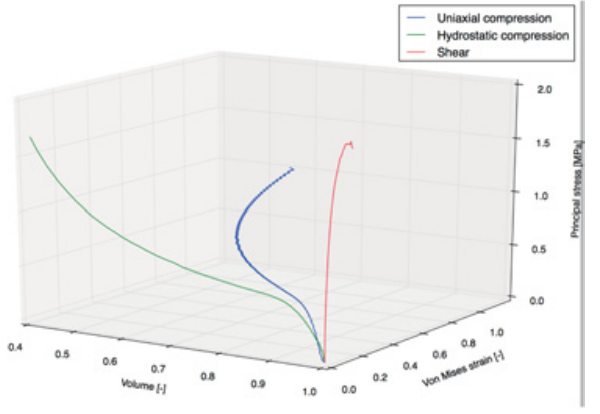

Figure 11. Three dimensional representation of the three experiments. Evolution of the principal stress vs. the shape change (Von Mises strain $\varepsilon_{V M}$ ) and vs. the volume change (Vol. $V$ ).

It is therefore possible to plot in the three axis (Prinicpal stress, Volume and Von Mises strain) the evolutions of each experiment, see Fig. 11. The hydrostatic compressive test and the shear test do not depend of the shape change and the volume change, respectively. Concerning the evolution of the uniaxial compressive test, it can be noted that at the beginning of the test, the foam is mainly subjected to volume change and progressively, the contribution of shape change increases. With these considerations, we can say that the shape change is related to the pressure level inside the foam and the modeling development must take into account this key point.

\section{Modeling}

\subsection{HVH model}

The numerical model used to describe foam behavior is the Hyperelastic - Viscous - Hysteretic (HVH) model. It has been used successfully to describe the behavior of materials such as shape memory materials NiTi [11], elastomeric materials [6] or polypropylene materials [12]. This model is based on the addition of three powers $\mathcal{P}_{\text {hyp }}$, $\mathcal{P}_{\text {vis }}$ and $\mathcal{P}_{\text {hys }}$ describing the three behavior contributions:

$$
\begin{aligned}
& \mathcal{P}_{\text {int }}=\mathcal{P}_{\text {hyp }}+\mathcal{P}_{\text {vis }}+\mathcal{P}_{\text {hys }} \\
& \int_{\mathcal{D}} \overline{\bar{\sigma}}: \overline{\bar{D}} d V=\int_{\mathcal{D}}\left(\overline{\bar{\sigma}}_{\text {hyp }}+\overline{\bar{\sigma}}_{\text {vis }}+\overline{\bar{\sigma}}_{\text {hys }}\right): \overline{\bar{D}} d V
\end{aligned}
$$

where $\mathcal{P}_{\text {int }}$ is the internal power, $\overline{\bar{\sigma}}_{\text {hyp }}, \overline{\bar{\sigma}}_{\text {vis }}$ and $\overline{\bar{\sigma}}_{\text {hys }}$ are the hyperelastic, the viscous and the hysteretic stress tensor, $\overline{\bar{D}}$ is the strain rate tensor and $\mathcal{D}$ the studied domain.

The viscous model chosen for this study is the classical generalized Maxwell's model composed of $n$ springdashpot in parallel. The parameters of the model can be identified by relaxation tests. The hysteretic model is the one used in the research works of Rio, Favier et al., for example in [11]. The hysteretic stress $\sigma_{\text {hys }}$ is assumed to be purely deviatoric and irreversible. These two previous contributions do not concern the topic of this article and readers can find more explanations about the hysteretic model in $[11,13,14]$.

The hyperelastic contribution is developed by treating individually the spherical and the deviatoric behaviors in order to take into account the differences between the shape change and the volume change behaviors in cellular materials. The hyperelastic potential $\omega_{\text {hyp }}$ can be written as the contribution of a spherical $\omega_{\text {hyp }}^{s p h}$ and a deviatoric $\omega_{\text {hyp }}^{\text {dev }}$ contribution:

$$
\omega_{h y p}=\omega_{h y p}^{s p h}+\omega_{h y p}^{d e v} .
$$

\subsection{Hyperelastic model - spherical behavior}

The spherical contribution of the hyper elastic model is only related to the volume change $V$. With a phenomenological approach, it is possible to describe hydrostatic behavior using a polynomial form of potential equation which check (i) the positivity of the potential over the volume change and (ii) the null-value of the potential for null-volume change, i.e. $V=1$. With these considerations, a hyperelastic potential is proposed:

$$
\omega_{\text {hyp }}^{s p h}(V)=a_{i} V^{i}(V-1)^{2}
$$

where $a_{i}$ are the parameters of the $i$-order polynom. The pressure can be obtained easily from the potential. The free energy $E$ can be obtained by:

$$
E=\int_{\mathcal{D}} \omega d V=\int_{\mathcal{D}_{\text {ref }}} \omega \sqrt{g} d \theta^{i}
$$

where $g$ is the determinant of the metric tensor and $\theta_{i}$ the material coordinates. The power $\dot{E}$ can be calculated using classical equations of continuum mechanics with the Cauchy stress tensor $\overline{\bar{\sigma}}$ and the strain rate tensor $\overline{\bar{D}}$ based on the Almansi's strain:

$$
\dot{E}=\int_{\mathcal{D}_{\text {ref }}} \frac{d(\omega \sqrt{g})}{d t} d \theta^{i}=\int_{\mathcal{D}_{\text {ref }}} \overline{\bar{\sigma}}: \overline{\bar{D}} \sqrt{g} d \theta^{i} .
$$

This involves that:

$$
\overline{\bar{\sigma}}: \overline{\bar{D}}=\frac{1}{\sqrt{g}} \frac{\partial(\omega \sqrt{g})}{\partial t} .
$$

In the case of an hydrostatic compressive test,

$$
\overline{\bar{\sigma}}: \overline{\bar{D}}=-p \mathbf{I}_{\overline{\bar{D}}}=-p \frac{\dot{V}}{V}
$$

where $\mathbf{I}_{\bar{D}}$ is the first invariant, i.e. the trace, of the tensor $\overline{\bar{D}}$. By developing equation 6 with $V=\sqrt{g} / \sqrt{g_{0}}$ and using Eq. (7), it finally comes:

$$
-p=V \frac{\partial \omega}{\partial V}+\omega
$$

It is therefore possible to express the pressure $p(V)$ with the $a_{i}$ parameters of the model and for a $4^{\text {th }}$-order polynom, it gives for example:

$$
\begin{aligned}
& \omega(V)= a_{0}+\left(-2 a_{0}+a_{1}\right) V^{1}+\left(a_{0}-2 a_{1}+a_{2}\right) V^{2} \\
&+\left(a_{1}-\right.\left.2 a_{2}+a_{3}\right) V^{3}+\left(a_{2}-2 a_{3}+a_{4}\right) V^{4} \\
&+\left(a_{3}-2 a_{4}\right) V^{5}+\left(a_{4}\right) V^{6} \\
&-p(V)= a_{0}+2\left(-2 a_{0}+a_{1}\right) V^{1}+3\left(a_{0}-2 a_{1}+a_{2}\right) V^{2} \\
&+4\left(a_{1}-2 a_{2}+a_{3}\right) V^{3}+5\left(a_{2}-2 a_{3}+a_{4}\right) V^{4} \\
&+6\left(a_{3}-2 a_{4}\right) V^{5}+7\left(a_{4}\right) V^{6}
\end{aligned}
$$




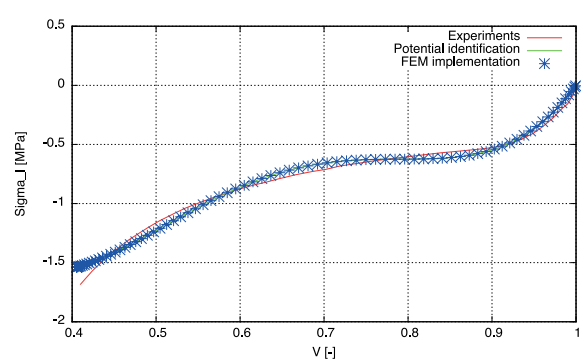

Figure 12. Spherical potential $\omega_{h y p}^{s p h}$ identification thanks to equation 11 and FEM implementation validation in Herezh++ [15].

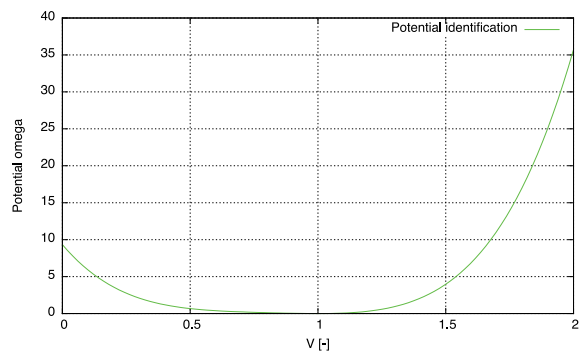

Figure 13. Spherical potential $\omega_{\text {hyp }}^{s p h}$ shape.

The determination of the spherical model parameters becomes easy with the results of an hydrostatic compressive test. The Figs. 12 and 13 show the identified model compared to experimental results and the shape of the spherical potential.

The use of a polynomial function for the spherical hyperelastic potential is quite accurate to model spherical behavior (see Fig. 12). This potential check the thermodynamic conditions with a positive value of $\omega_{\text {hyp }}^{s p h}>$ $0 \forall V$ and a null-value of $\omega_{\text {hyp }}^{s p h}=0 \Leftrightarrow V=1$ (see Fig. 13).

\subsection{Hyperelastic model - deviatoric behavior}

Many deviatoric potentials exist and can be used for hyperelastic behavior. The Money-Rivlin [16,17] and Ogden [18] models are the famous ones. These potentials are based on the invariants of the Cauchy-Green stress tensor $\overline{\bar{B}}$ as the Mooney-Rivlin (MR) one:

$$
\omega_{M R}\left(\mathbf{I}_{\overline{\bar{B}}}, \mathbf{I I}_{\overline{\bar{B}}}, \mathbf{I I I}_{\overline{\bar{B}}}\right)=C_{10}\left(\overline{\mathbf{I}}_{\overline{\bar{B}}}-3\right)+C_{01}\left(\overline{\mathbf{I}}_{\overline{\bar{B}}}-3\right)
$$

with:

$$
\begin{aligned}
\mathbf{I}_{\overline{\bar{B}}} & =\lambda_{1}^{2}+\lambda_{2}^{2}+\lambda_{3}^{2} \\
\mathbf{I I}_{\overline{\bar{B}}} & =\lambda_{1}^{2} \lambda_{2}^{2}+\lambda_{1}^{2} \lambda_{3}^{2}+\lambda_{2}^{2} \lambda_{3}^{2} \\
\mathbf{I I I}_{\overline{\bar{B}}} & =\lambda_{1}^{2} \lambda_{2}^{2} \lambda_{3}^{2}
\end{aligned}
$$

where $\lambda_{i}$ are the stretches of the Cauchy-Green tensor and:

$$
\overline{\mathbf{I}}_{\overline{\bar{B}}}=\mathbf{I}_{\overline{\bar{B}}} \cdot \mathbf{I I I}_{\overline{\bar{B}}}^{-\frac{1}{3}} \quad \text { and } \quad \overline{\mathbf{I}}_{\overline{\bar{B}}}=\mathbf{I I}_{\overline{\bar{B}}} \cdot \mathbf{I I I} \overline{\overline{\bar{B}}}_{\overline{\bar{B}}}^{-\frac{2}{3}} \text {. }
$$

The use of the Cauchy-Green stress tensor is a good way to separate shape change and volume change. However, the parameters of the MR potential are difficult to identify directly and moreover in a case of shear test. Indeed, the

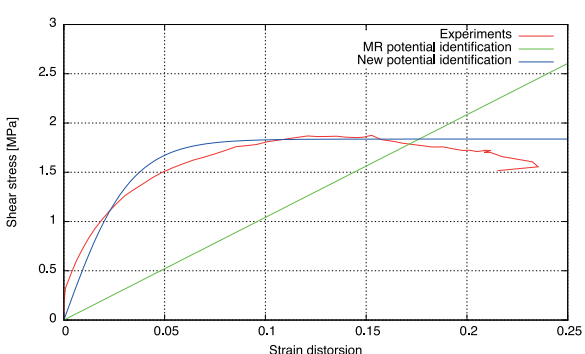

Figure 14. Deviatoric potential $\omega_{h y p}^{s p h}$ identification thanks to Eq. (18).

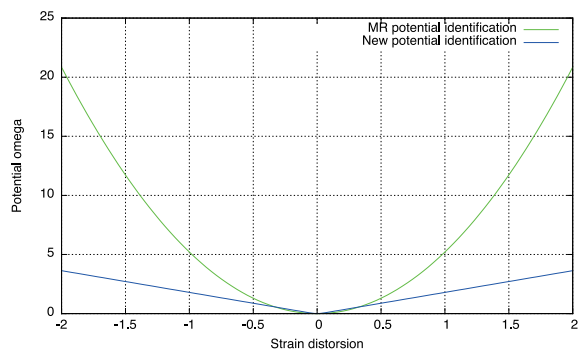

Figure 15. Deviatoric potential $\omega_{\text {hyp }}^{\text {dev }}$ shape.

shear test involves these three principal stretches:

$$
\lambda_{1,2}^{2}=\left(1+\frac{\gamma^{2}}{2}\right) \pm\left(\gamma \sqrt{1+\frac{\gamma^{2}}{4}}\right) \quad \text { and } \quad \lambda_{3}^{2}=1
$$

with $\gamma$ is the strain distorsion and the invariants can be calculated as:

$$
\overline{\mathbf{I}}_{\overline{\bar{B}}}=\overline{\mathbf{I}}_{\overline{\bar{B}}}=\lambda^{2}+\frac{1}{\lambda^{2}}+1
$$

which leads to an indetermination to identify separately the $C_{10}$ and the $C_{01}$ parameters. With these considerations, a new model based on the potential of Favier used in [6] and using the Cauchy-Green stress tensor invariants has been proposed.

$\omega\left(\overline{\mathbf{I}}_{\overline{\bar{B}}}\right)=\frac{Q_{\sigma}^{2}}{\mu_{1}} \ln \left[\cosh \left(\frac{\mu_{1}}{Q_{\sigma}} \sqrt{\left(\overline{\mathbf{I}}_{\overline{\bar{B}}}-3\right)}\right)\right]+\mu_{2}\left(\overline{\mathbf{I}}_{\overline{\bar{B}}}-3\right)$

with $Q_{\sigma}, \mu_{1}$ and $\mu_{2}$ the three parameters of the deviatoric potential.

It is therefore possible to calculate the stress from the expression of the deviatoric tensor:

$$
\sigma^{i j}=\frac{\partial \omega}{\partial \varepsilon_{A i j}}=\frac{\partial \omega}{\partial \overline{\mathbf{I}}_{\overline{\bar{B}}}} \frac{\partial \overline{\mathbf{I}}_{\overline{\bar{B}}}}{\partial \gamma} \frac{\partial \gamma}{\partial \varepsilon_{A i j}}
$$

and gives:

$$
\sigma^{12}=Q_{\sigma} \tanh \left(\frac{\mu_{1}}{Q_{\sigma}} \gamma\right)+2 \mu_{2} \gamma
$$

The three parameters of the deviatoric potential has been identified with $\mu_{1}=56.2 \mathrm{MPa}, Q_{\sigma}=1.84 \mathrm{MPa}$ and $\mu_{2}$ has been fixed to $0 \mathrm{MPa}$ in order to prevent the potential 
from being negative. Results are presented in Figs. 14 and 15. New potential identification can be performed easily compared with MR potential because the value of $\mu_{1}+\mu_{2}$ is the slope of the first part of the identified curve, $\mu_{2}$ is the slope of the second part of the identified curve and $Q_{\sigma}$ is the intersection of the second slope and the ordinate axis.

\section{Conclusion}

This research work proposed to study a PP foam by performing some experiments to exhibit its volume change and its shape change by using hydrostatic compressive test and shear test, respectively. The dependance of pressure level for cellular materials leads the authors to develop an original combined pressure-shear test using an hexapod device.

Finite Element modeling must take into account spherical and deviatoric contributions separately. With these considerations, two Hyperelastic models have been proposed and studied. The first spherical one is based on a polynomial function and can be easy identified from a hydrostatic compressive test. The second deviatoric one is based on the first invariant of the Cauchy-Green stress tensor and can also be easy to identify using a shear test.

Further works will focus on the combined compressiveshear test to get the shape change under different pressure level. These experiments will give informations about the coupling between shape change and volume change. Hyperelastic model will be therefore adapted.

\section{References}

[1] N.J. Mills, C. Fitzgerald, A. Gilchrist, R. Verdejo. Compos Sci Technol 63(16), 2389-2400 (2003)

[2] O. Hallquist. LS-Dyna3D theoritical manual, Livermore Software Technology (1998)
[3] A.G. Hanssen, O.S. Hopperstad, M. Langseth, H. Ilstad. Int J Mech Sci 44(2), 359-406 (2002)

[4] P. Viot. International Journal Impact Engineering 36, 975-989 (2009)

[5] E. Combaz, C. Bacciarini, R. Charvet, W. Dufour, F. Dauphin, A. Mortensen. Acta Materialia 58, 5168-5183 (2010)

[6] A. Vandenbroucke, H. Laurent, N. Ait Hocine, G. Rio. Computational Materials Science 48, 495-503 (2010)

[7] P. Viot, F. Beani, J.-L. Lataillade. J Mater Sci 40, 5829-5837 (2005)

[8] R. Bouix, P. Viot, J.-L. Lataillade. Int J Imp Eng 36, 329-342 (2009)

[9] L. Maheo, P. Viot. Int J Imp Eng 53, 84-93 (2013)

[10] S. Bouvier, H. Haddadi, P. Levée, C. Teodosiu. J Mater Proc Technol 172(1), 96-103 (2006)

[11] B.S. Shariat, Y. Liu, G. Rio. J Intel Mater Syst Struct 25(12), 1445-1455 (2014)

[12] M. Zrida, H. Laurent, G. Rio, S. Pimbert, V. Grolleau, N. Masmoudi, C. Bradai. Comput Mater Sci 45(2), 516-527 (2009)

[13] P. Guélin. J Mécanique 19(2), 217-247 (1980)

[14] P. Pegon. PhD thesis, Institut National Polytechnique, Université Joseph Fourier, Grenoble (1988)

[15] G. Rio. Herezh++: FEM software for large transformations in solids. Dépôt APP - Certification IDDN.FR.010.0106078.000.R.P.2006.035.20600, LIMATB-UBS (2015)

[16] M. Monney. J Appl Phys 11(9), 582-592 (1940)

[17] R.S. Rivlin. Philos Trans Roy Soc London Ser A? Math Phys Eng Sci A 241(835), 379-397 (1948)

[18] R.W. Ogden. Mineola (NY): Dover Publication Inc. (1984) 